\title{
On addition of two sets of integers
}

\author{
by \\ Yong-Gao Chen (Nanjing)
}

1. Introduction. Let $A=\left\{a_{1}, \ldots, a_{k}\right\}$ and $B=\left\{b_{1}, \ldots, b_{l}\right\}$ be two sets of integers with $k=|A|, l=|B|$ and $0=a_{1}<\ldots<a_{k}, 0=b_{1}<\ldots<b_{l}$. Denote the set $\left\{a_{i}+b_{j}: 1 \leq i \leq k, 1 \leq j \leq l\right\}$ by $A+B$ and $A+A$ by $2 A$. Assume that $a_{k} \geq b_{l}$.

G. Freiman [1] showed

TheOREM 1. (i) Let $a_{k} \leq 2 k-3$. Then $|2 A| \geq a_{k}+k$.

(ii) Let $a_{k} \geq 2 k-2$ and $\left(a_{1}, \ldots, a_{k}\right)=1$. Then $|2 A| \geq 3 k-3$.

G. Freiman [2] generalized this to the case of two distinct summands.

TheOREM 2. (i) Let $a_{k} \leq k+l-3$. Then $|A+B| \geq a_{k}+l$.

(ii) Let $a_{k} \geq k+l-2$ and $\left(a_{1}, \ldots, a_{k}, b_{1}, \ldots, b_{l}\right)=1$. Then $|A+B| \geq$ $k+l+\min \{k, l\}-3$.

Let

$$
\delta= \begin{cases}1 & \text { if } b_{l}=a_{k} \\ 0 & \text { if } b_{l} \neq a_{k}\end{cases}
$$

Vsevolod F. Lev and Pavel Y. Smeliansky [5] sharpened the above Theorem 2 as

Theorem 3. (i) Let $a_{k} \leq k+l-2-\delta$. Then $|A+B| \geq a_{k}+l$.

(ii) Let $a_{k} \geq k+l-1-\delta$ and $\left(a_{1}, \ldots, a_{k}\right)=1$. Then $|A+B| \geq k+2 l-2-\delta$.

In this paper we further sharpen the above Theorem 3 . The main results are given in the next section.

2. Statements of main results. In the following we do not use the notations of the first section except in the Corollary of Theorem 4. For any set $T$ of integers, let $|T|$ denote the cardinality of $T$, and $d_{T}$ denote the greatest common divisor of elements of $T-\{t\}$, where $t \in T$. It is not difficult

The subject was supported by Fok Ying Tung Education Foundation. This work was done during my visiting Institute of Mathematics, Academia Sinica. 
to see that $d_{T}$ is independent of the choice of $t$. Let $q$ be a positive integer. We use $\bar{T}$ to denote the set of residue classes modulo $q$ having nonempty intersection with $T$. Let $A, B$ be two nonempty finite sets of integers and write

$$
\begin{aligned}
& A=\bigcup_{i=1}^{t} A_{i}, \quad\left|\bar{A}_{i}\right|=1, \quad \bar{A}_{i} \cap \bar{A}_{j}=\emptyset, \quad i \neq j ; \\
& B=\bigcup_{i=1}^{s} B_{i}, \quad\left|\bar{B}_{i}\right|=1, \quad \bar{B}_{i} \cap \bar{B}_{j}=\emptyset, \quad i \neq j .
\end{aligned}
$$

Then $|\bar{A}|=t$ and $|\bar{B}|=s$. In this paper we prove

Theorem 4. (i) Let $q \leq|\bar{A}|+|\bar{B}|-1$. Then

$$
|A+B| \geq q+|B|+\left(\max _{i}\left|A_{i}\right|-2\right)|\bar{B}| \text {. }
$$

(ii) Let $q \geq|\bar{A}|+|\bar{B}|$ and $\left(d_{A}, d_{B}, q\right)=1$. Then

$$
|A+B| \geq \min \left\{\frac{2 q}{\left(q, d_{A}\right)},|\bar{A}|+|\bar{B}|-1\right\}+|B|+\left(\max _{i}\left|A_{i}\right|-2\right)|\bar{B}| .
$$

From Theorem 4 we immediately have

Corollary. Let the notations be as in Section 1. Then

(i) if $a_{k} \leq k+l-2-\delta$, then

$$
|A+B| \geq a_{k}+l
$$

(ii) if $a_{k} \geq k+l-1-\delta$ and $\left(a_{1}, \ldots, a_{k}, b_{1}, \ldots, b_{l}\right)=1$, then

$$
|A+B| \geq \min \left\{\frac{2 a_{k}}{d_{A}}, k+l-2-\delta\right\}+l .
$$

Note. Theorem 4 is sharp. For example, let

$$
\begin{aligned}
& A=\{n: n \equiv 1,2(\bmod 5), 1 \leq n \leq 5 k\}, \\
& B=\{m: m \equiv 0,3,4(\bmod 5), 1 \leq m \leq 5 k\} .
\end{aligned}
$$

Then

$$
A+B=\{n: n \equiv 0,1,2,4(\bmod 5), 3 \leq n \leq 10 k-2\} .
$$

So $|A+B|=8 k-4$. Now we use Theorem 4 . Take $q=5 k-4$. Then $|\bar{A}|=2 k-1,|\bar{B}|=3 k-2,|B|=3 k$ and $\max _{i}\left|A_{i}\right|=2$. By Theorem 4 we have

$$
|A+B| \geq \min \{5 k-4,5 k-4\}+3 k=8 k-4 .
$$

\section{Proof of the main results}

Lemma 1. Let $T$ be a set of integers, and $T_{1}$ a subset of $T$. Then

$$
|T|-|\bar{T}| \geq\left|T_{1}\right|-\left|\bar{T}_{1}\right| .
$$


Proof. Write $T=T_{1} \cup T_{2}, T_{1} \cap T_{2}=\emptyset$. Then

$$
|T|=\left|T_{1}\right|+\left|T_{2}\right|, \quad|\bar{T}|=\left|\bar{T}_{1} \cup \bar{T}_{2}\right|=\left|\bar{T}_{1}\right|+\left|\bar{T}_{2}\right|-\left|\bar{T}_{1} \cap \bar{T}_{2}\right| .
$$

Hence

$$
|T|-|\bar{T}|=\left|T_{1}\right|-\left|\bar{T}_{1}\right|+\left|T_{2}\right|-\left|\bar{T}_{2}\right|+\left|\bar{T}_{1} \cap \bar{T}_{2}\right| \geq\left|T_{1}\right|-\left|\bar{T}_{1}\right| .
$$

This completes the proof of Lemma 1.

LEMma 2. We have

$$
\left|A_{i}+B\right| \geq\left|\bar{A}_{i}+\bar{B}\right|+|B|+\left(\left|A_{i}\right|-2\right)|\bar{B}|, \quad i=1, \ldots, t .
$$

Proof. Since $\bar{A}_{i}+\bar{B}_{1}, \bar{A}_{i}+\bar{B}_{2}, \ldots, \bar{A}_{i}+\bar{B}_{s}$ are pairwise disjoint, we have

$$
\left|\bar{A}_{i}+\bar{B}\right|=\sum_{j=1}^{s}\left|\bar{A}_{i}+\bar{B}_{j}\right|=s
$$

and

$$
\left|A_{i}+B\right|=\sum_{j=1}^{s}\left|A_{i}+B_{j}\right| \geq \sum_{j=1}^{s}\left(\left|A_{i}\right|+\left|B_{j}\right|-1\right)=s\left(\left|A_{i}\right|-1\right)+|B| .
$$

By $|\bar{B}|=s$ Lemma 2 is true.

Lemma 3 (Kneser's Theorem [3], [4]). Let $H$ be the subgroup of all those elements $h \in \mathbb{Z}_{q}$ satisfying $\bar{A}+\bar{B}+h=\bar{A}+\bar{B}$. If

$$
|\bar{A}+\bar{B}| \leq|\bar{A}|+|\bar{B}|-1,
$$

then

$$
|\bar{A}+\bar{B}|+|H|=|\bar{A}+H|+|\bar{B}+H| .
$$

Hence $|H|>1$ if

$$
|\bar{A}+\bar{B}| \leq|\bar{A}|+|\bar{B}|-2 .
$$

Proof of Theorem 4 (following the proof of Lev and Smeliansky [5]). Without loss of generality, we may assume that $0 \in A, 0 \in B$ and $\max _{i}\left|A_{i}\right|=\left|A_{1}\right|, \bar{A}_{1}=\{0\}$. Suppose that

$$
|A+B| \leq|\bar{A}|+|B|+\left(\left|A_{1}\right|-1\right)|\bar{B}|-2
$$

and prove that

$$
\begin{aligned}
|A+B| \geq q+|B|+\left(\left|A_{1}\right|-2\right)|\bar{B}| & \text { if } q \leq|\bar{A}|+|\bar{B}|-1 ; \\
|A+B| \geq \min \left\{\frac{2 q}{\left(q, d_{A}\right)}, q\right\}+|B|+ & \left(\left|A_{1}\right|-2\right)|\bar{B}| \\
& \text { if } q \geq|\bar{A}|+|\bar{B}| \text { and }\left(d_{A}, d_{B}, q\right)=1 .
\end{aligned}
$$

By Lemmas 1 and 2 we have 


$$
|A+B|-|\bar{A}+\bar{B}| \geq\left|A_{1}+B\right|-\left|\bar{A}_{1}+\bar{B}\right| \geq|B|+\left(\left|A_{1}\right|-2\right)|\bar{B}| .
$$

So it suffices to prove that

$$
\begin{gathered}
|\bar{A}+\bar{B}|=q \quad \text { if } q \leq|\bar{A}|+|\bar{B}|-1 \\
|\bar{A}+\bar{B}| \geq \min \left\{\frac{2 q}{\left(q, d_{A}\right)}, q\right\} \quad \text { if } q \geq|\bar{A}|+|\bar{B}| \text { and }\left(d_{A}, d_{B}, q\right)=1 .
\end{gathered}
$$

By (1) and (2) we have

$$
|\bar{A}+\bar{B}| \leq|\bar{A}|+|\bar{B}|-2 .
$$

By Lemma 3 we have $|H|>1$. Then $H=d \mathbb{Z}_{q}, d \mid q, d>0$. Let $\phi: \mathbb{Z} \rightarrow \mathbb{Z}_{q}$ be the canonical homomorphism of $\mathbb{Z}$ onto $\mathbb{Z}_{q}$, and $\sigma: \mathbb{Z}_{q} \rightarrow \mathbb{Z}_{q} / H$ the canonical homomorphism. Let $\mathcal{A}=\sigma(\phi A)$ and $\mathcal{B}=\sigma(\phi B)$. If $d=1$ then $\bar{A}+\bar{B}=\mathbb{Z}_{q}$. In the following we assume that $d>1$. By Lemmas 1 and 2 we have

$$
\begin{aligned}
\mid\{c \in A & +B: \phi c \in \bar{B}+H\}|-|\{\bar{c} \in \bar{A}+\bar{B}: \bar{c} \in \bar{B}+H\} \mid \\
& \geq\left|\left\{c \in A_{1}+B: \phi c \in \bar{B}+H\right\}\right|-\left|\left\{\bar{c} \in \bar{A}_{1}+\bar{B}: \bar{c} \in \bar{B}+H\right\}\right| \\
& =\left|\left\{c \in A_{1}+B\right\}\right|-\left|\left\{\bar{c} \in \bar{A}_{1}+\bar{B}\right\}\right| \geq|B|+\left(\left|A_{1}\right|-2\right)|\bar{B}| .
\end{aligned}
$$

As in [5], we may derive that $\mathcal{A}=\{0\}$, that is, $\bar{A} \subseteq H$. So $|\bar{A}| \leq|H|=q / d$, that is, $q \geq d|\bar{A}|$. We also have

$$
|\bar{B}| \leq|\bar{B}+H|=|\mathcal{B}| \cdot|H|=|\mathcal{B}| q / d .
$$

If $q \leq|\bar{A}|+|\bar{B}|-1$, then

$$
q \leq|\bar{A}|+|\mathcal{B}| \frac{q}{d}-1 .
$$

That is, $q(d-|\mathcal{B}|) \leq d(|\bar{A}|-1)$. So $d|\bar{A}|(d-|\mathcal{B}|) \leq d(|\bar{A}|-1)$. Hence $d=|\mathcal{B}|$. Therefore

$$
|\bar{A}+\bar{B}|=|\bar{A}+\bar{B}+H|=|\bar{B}+H|=|\mathcal{B}| \frac{q}{d}=q .
$$

Since $\mathcal{A}=\{0\}$, we have $d \mid d_{A}$, whence $d \mid\left(d_{A}, q\right)$. If $q \geq|\bar{A}|+|\bar{B}|$ and $\left(d_{A}, d_{B}, q\right)=1$, from $d>1$ and $\mathcal{A}=\{0\}$ we have $\mathcal{B} \neq\{0\}$, whence $|\mathcal{B}| \geq 2$. Hence

$$
|\bar{A}+\bar{B}|=|\bar{A}+\bar{B}+H| \geq|\bar{B}+H|=|\mathcal{B}| \frac{q}{d} \geq \frac{2 q}{d} \geq \frac{2 q}{\left(d_{A}, q\right)} .
$$

This completes the proof of Theorem 4 .

Remark. Let $\left(d_{A}, d_{B}, q\right)=1$. By the same method we may prove that

$$
\begin{aligned}
|A+B| \geq & \min \{q,|\bar{A}|+|\bar{B}|-1\} \\
& +\min \left\{|B|+\left(\max _{i}\left|A_{i}\right|-2\right)|\bar{B}|,|A|+\left(\max _{i}\left|B_{i}\right|-2\right)|\bar{A}|\right\} .
\end{aligned}
$$




\section{References}

[1] G. Freiman, On addition of finite sets, I, Izv. Vyssh. Uchebn. Zaved. Mat. 1959 (6), 203-213.

[2] -, Inverse problems of additive number theory, VI, ibid. 1962 (3), 151-157.

[3] M. Kneser, Abschätzung der asymptotischen Dichte von Summenmengen, Math. Z. 58 (1953), 459-484.

[4] - Ein Satz über abelschen Gruppen mit Anwendungen auf die Geometrie der Zahlen, ibid. 61 (1955), 429-434.

[5] V. F. Lev and P. Y. Smeliansky, On addition of two distinct sets of integers, Acta Arith. 70 (1995), 85-91.

Department of Mathematics Nanjing Normal University

Nanjing 210097, Jiangsu

P.R. China 